\title{
Genus-Zuweisung bei der Pronominalisierung von Personen in den Südwalser Dialekten*
}

\author{
Peter Zürrer (Zürich)
}

\begin{abstract}
The Alemannic dialects in linguistic islands in Northern Italy have been undergoing strong changes since the second half of the 20th century. One of these changes concerns the assignment of gender with persons. Generalized neuter abolishes the coupling of gender with male/female and transfers both female and male persons into neuter. This in turn has its effect on verbal inflection. The post-verbal subject clitics mutate in the $3 \mathrm{rd}$ person singular to verb endings void of male/female connotations. This change, as it is now spreading, is not in itself a recent phenomenon. In early written dialectal records it already occurs in single documents even at the beginning of the 19th century.
\end{abstract}

\section{Einleitung}

\subsection{Vorausschau}

Sprachinseldialekte - und um solche handelt es sich bei den Südwalser Mundarten - bewahren in der Regel altertümliche Züge und übernehmen im Sprachkontakt fremde Strukturen, vor allem aber gehen sie eigene Wege, und dies ist der Fall bei unserem Thema, der Genus-Zuweisung. Die Pronominalisierung von Personen und Personenbezeichnungen weicht hier erheblich ab von binnendeutschen und schweizerdeutschen Verhältnissen, und selbst mit den benachbarten Walliser Dialekten stimmt sie nicht überein; dasselbe gilt für die präonymische ArtikelwortZuteilung bei Personennamen. Direkter romanischer Einfluss ist nicht nachzuweisen, ebenso wenig Reliktstellung; es handelt sich vielmehr um eine auffällige Eigenentwicklung, die zwar schon angelegt war und fürs vorletzte Jahrhundert nachgewiesen werden kann, die sich aber erst im ausgehenden letzten Jahrhundert ausweitete und sich seither verallgemeinert.

Vereinfacht lässt sich festhalten: Die Pronominalisierung favorisiert in unvorhergesehenem Masse die Zuweisung des Neutrums - Pronominalisierung ist im Wesentlichen Neutralisierung. Besonders hervorzuheben ist, dass sich die Neutrum-Zuweisung nicht auf weibliche Personen

\footnotetext{
* Überarbeitete und erweiterte Fassung des Kapitels „Geschlechtsneutralisierung bei der Pronominalisierung von Personen“ in Zürrer (1999: 245-256). Die Materialien stammen aus eigenen Befragungen bzw. eigenen (Ton-) Aufnahmen oder aus publizierten Transkriptionen anderer Autoren (in vereinfachter bzw. angepasster Schreibung wiedergegeben). - Für hilfreiche Hinweise danke ich Damaris Nübling und Harald Burger.
}

Linguistik online 107, 2/21 - http://dx.doi.org/10.13092/lo.107.7694

CC by 3.0 
und Personenbezeichnungen beschränkt, sondern gleicherweise Personen männlichen Geschlechts betrifft. Folgerichtig werden auch die männlichen wie die weiblichen Vollnamen und nicht nur die Diminutive - übereinstimmend mit neutralen Artikelformen versehen. Das Neutrum ist in den Südwalser Mundarten nicht das für exophorische Personenbezüge, Personenbezeichnungen und Personennamen am wenigsten (cf. Nübling 2017: 180f.), sondern das am meisten genutzte Genus, es tendiert zum Genus für Personen schlechthin.

Die Dinge sind freilich im Fluss (und insofern bedarf die genannte „Vereinfachung“ einer Ergänzung). Die Genus-Zuweisung befindet sich trotz dem Vormarsch der Neutra in einer Übergangsphase, wo die herkömmliche, soziopragmatische Maskulin-Feminin-Neutrum-Zuweisung immer noch, wenn auch stark eingeschränkt, Gültigkeit hat.

\subsection{Zur äusseren Sprachsituation}

Wenn wir die Verbreitung der Südwalser Dialekte in den Blick nehmen, fällt ein arealtypologischer Gegensatz auf: Wir sind nicht mit flächendeckenden Dialekt-Arealen innerhalb des deutschen Sprachgebiets konfrontiert, sondern mit isolierten Siedlungen an dessen Rand. Topographisch und politisch sind sie zersplittert, sie gehören zu Italiens Regionen Aostatal und Piemont und zum schweizerischen Kanton Tessin. Es sind Sprachinseln, vom zusammenhängenden deutschen Sprachgebiet getrennt durch die Alpenkette, durch Viertausender wie das Monte-Rosa-Massiv; es sind - mit Ausnahme von Issime in der Region Aostatal - Ortsgemeinden in Hanglage oder am Ende von langen, engen Tälern, ohne Verkehrsverbindung untereinander, es sei denn über heute nicht mehr begangene Fusspässe. Auch zum Wallis, aus dem die Walser im Hochmittelalter emigriert sind, bestehen keine Verbindungen mehr.

Die Orte sind heute so gut wie italienischsprachig; der Walser Dialekt wird ausschliesslich ortsintern gesprochen und auch hier nurmehr von einer Minderheit; dessen Gebrauch ist mehr oder weniger eingeschränkt auf familiären Umgang. ${ }^{1}$

Unter sozial- und wirtschaftsgeschichtlichem Aspekt hatten die Südwalser (und insbesondere die Gressoneyer) eine Sonderstellung im Vergleich mit den Walsern in Graubünden und Vorarlberg: Sie hängt zusammen mit der zentralen Rolle der Frau im bergbäuerlichen Produktionsprozess. Die Führung der Südwalser Bauernbetriebe lag in den Händen der Bäuerinnen. Es waren die Frauen, die die Wirtschaft führten: Sie setzten die Termine fest, sie wählten die Knechte und Mäher aus, sie stellten sie ein und bezahlten sie. Ihre Rolle ist zurückzuführen auf die seit Jahrhunderten in den Walser Südorten praktizierte Saisonauswanderung der Männer und deren daraus entstandene monatelange Abwesenheit. Diese geschlechtsspezifische Rollenverteilung galt bis zum Ersten Weltkrieg, zum Teil auch noch darüber hinaus.

\footnotetext{
${ }^{1}$ Sprachstatistische Angaben zu Gressoney und Issime in Zürrer (1999: 49-56, 67f.; 2009: 47-55, 69), zu allen Südwalser Ortsgemeinschaften (1999: 69, 386; 2009: 70f.).
} 


\section{Zuweisungsprinzipien}

\subsection{Maskulin-Feminin-Neutrum-Zuweisung: soziopragmatisch gesteuert ${ }^{2}$}

$\mathrm{Ob}$ das Genus gemäss dem natürlichen Geschlecht zugewiesen wird oder die Genus-SexusKongruenz aufgehoben wird, hängt vom Faktor ,,soziale Beziehung“ ab. Relative Nähe oder Distanz bzw. Symmetrie oder Asymmetrie im Verhältnis der Sprecherinstanz zur Referenzperson entscheidet über die Genuszuteilung. Und zwar wie folgt:

- Bei sozialer Distanz oder asymmetrischer Beziehung wird den Referenzpersonen das mit dem natürlichen Geschlecht korrespondierende Genus zugewiesen. Diese Regelung gilt bei der Pronominalisierung von Personen, die über Ansehen verfügen (Notar, Arzt, Lehrer, Gemeindesekretär, deren Ehefrauen, Pfarrer, weibliche Berufsleute wie Anwältinnen, Ärztinnen, Lehrerinnen). ${ }^{3}$ Bei Referenz auf Personen aus dem Familien- oder Bekanntenkreis, aus der Nachbarschaft, zu denen ein Altersabstand besteht und denen aus Konvention mit Respekt und höherer Achtung begegnet wird, gilt dieselbe sprachliche Regelung; bei Mutter, Vater, Grosseltern, Onkel und Tante ist sie freilich labil geworden und in schwankender, wenn nicht ausbleibender Anwendung. Die Regelung gilt ausserdem für den Bezug auf Ortsfremde (auch wenn man sie kennen gelernt hat), auf Feriengäste, Passanten, Beamte, Geschäftsleute, Unternehmer etc.

- Bei sozialer Nähe oder symmetrischer Beziehung wird von der Genuskorrespondenz zum natürlichen Geschlecht abgesehen, es gilt Neutralisierung. Gleiches Alter, gleicher Stand, verwandtschaftliche, nachbarschaftliche, freundschaftliche Beziehungen assoziieren sich mit Neutrumzuweisung.

Die Zuweisung des Genus folgt also soziopragmatischen Regeln. Die getroffene Genuswahl gewährt Aufschluss über das Beziehungsverhältnis zwischen Sprecher/Sprecherin und Referenzperson; das Genus erweist sich als Beziehungsanzeiger ${ }^{4}$.

\section{Beispiele für Maskulin-Feminin-Zuweisung auf Grund von Asymmetrie oder Distanz 5}

Mit (betonten) Vollformen (er, dschi (Gressoney), er, dschi/dschöi (Issime)): ${ }^{6}$

\footnotetext{
${ }^{2}$ Die Neutrumzuweisung und die damit verbundenen Kongruenzprobleme sind zum ersten Mal - mit Beschränkung auf die Verhältnisse in Rimella - ausführlicher dargestellt in Bauen (1978: 117-125). Zur Genuszuweisung in Issime und Gressoney cf. Zürrer (1999: 245-256), in Formazza cf. Dal Negro (2004: 154-158), in schweizerdeutschen Dialekten cf. Christen (1998: 271-280), im gesamtdeutschen und auf andere germanische Sprachen erweiterten Rahmen cf. Nübling (2017).

${ }^{3}$ Die Zuweisungsregel gilt von „unten“ nach „oben“. Wie sich umgekehrt höhergestellte Sprecher und Sprecherinnen auf tiefergestellte Referenzpersonen beziehen, ist kaum feststellbar, da jene heutzutage nicht mehr über den Dialekt verfügen und die Pronominaliserung auf Italienisch vornehmen.

${ }^{4}$ Zur beziehungsgesteuerten Genuszuweisung cf. Busley/Fritzinger (2018: 193-200), zu sozialer Distanz, Siezvs. Duz-Relation und weiteren genus-relevanten pragmatischen Faktoren cf. Nübling/Busley/Drenda (2013: 154, 164f., 193).

${ }^{5}$ Zur Zeichensetzung bei den angeführten Beispielen: Wörtliche Übersetzung wird in doppelte Anführungszeichen gesetzt, dabei wird je nach Verständnisschwierigkeit entweder das ganze Beispiel oder nur ein Teil übersetzt; die normale hochdeutsche Übersetzung steht in einfachen Anführungszeichen. Klitika werden mit dem Gleichheitszeichen, $=$, angeschlossen.

${ }^{6}$ Die hier angeführten Beispiele aus Issime und Gressoney (1-3) sind ausnahmsweise nicht frei formuliert, sondern wurden von den Gewährsleuten in einer Befragung aus dem Italienischen übersetzt.
} 
(1) woa ischt der engger? er ischt in der chilchu ,wo ist der Pfarrer? Er ist in der Kirche' (Issime)

(2) wer hät es gscheft uf em platz ghät? dschii, miin mamma hät eis ghät ,wer hat ein Geschäft im Dorfzentrum (,,auf dem Platz“) gehabt? $\underline{\text { Sie, }} \underline{\text { meine Mutter hat }}$ eines gehabt' (Gressoney)

(3) wüvil joar het dar oalt papa? er het aisunnöinzg ,wie alt ist der Grossvater? Er ist einundneunzig' (Issime)

Mit Klitika $(=e r,=d s c h,=s$, das Zeichen = bedeutet klitischen Anschluss):

(4) hit mö̈rgund ter hééru welt üsser chomo scheege t mäsch. isch=er passart en dum alter der

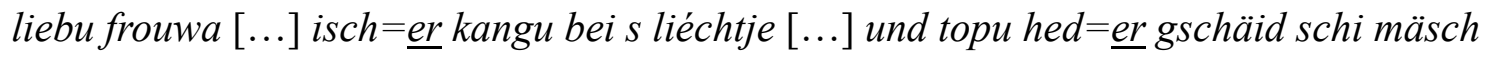
, heute morgen will der Pfarrer ausser Haus gehen, um die Messe zu lesen. Er ist am Altar der Mutter Gottes vorbeigegangen ... er ist zum Licht hingegangen ... und darauf hat er die Messe gelesen' (Rimella, Bauen 1978: 209)

(5) geit= $\underline{\text { er }}$ z ougschtal, de schirschang ,er geht nach Aosta, der $\underline{\text { Sergeant' }}^{‘}$ (Issime)

Beispiele für Neutrum-Zuweisung auf Grund von Symmetrie oder Nähe (Vollformen: ääs (Gressoney), iis (Issime), ees (Rimella) ${ }^{7}$, Klitika: $=i s,=e s,=s$ )

Bei dieser Zuweisung kann es zu grammatischer Inkongruenz kommen: Die soziopragmatische Forderung nach Geschlechtsneutralisierung gerät mit der Forderung nach Genusübereinstimmung mit dem substantivischen Bezugswort in Konflikt. Dies ist der Fall in den Beispielen (10, 11); die Problematik wird in Kapitel 5 wieder aufgegriffen.

(6) wer het=der treit der wii? $\underline{a ̈ a} s$

,wer hat dir den Wein gebracht? Er/sie“ („ess“, betont) (Gressoney)

(7) dass chemi schwetsen iis auch

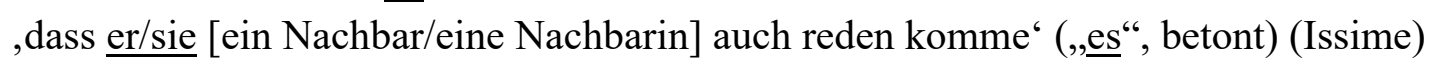

(8) tut $=\underline{\text { s }}$ der telefoné $\underline{\text { iis }}$

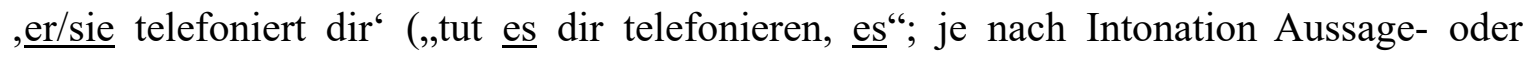
Fragesatz) (Issime)

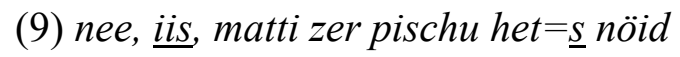

,nein, er/sie hat keine Wiesen in P.“'(„nein, es, Wiesen in P. hat es nicht“") (Issime)

(10) hit mim brioder chunt nit, ma schu hättet $=\underline{\underline{s}}$ zit schu chentet $=\underline{\underline{S}}$

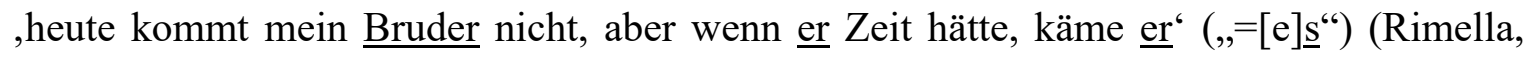
Bauen 1978: 118)

(11) diö schiwer gschi da bei d $\underline{\text { ajo }}$ wa hed $=\underline{s}$ gwäscht

,als wir dort bei der Mutter waren, wo sie $\left(,,=[\mathrm{e}] \underline{\mathrm{s}^{“}}\right)$ gewaschen hat" (Rimella, Bauen 1978: 119)

Beim präonymisch gesetzten Artikel zu weiblichen und männlichen Personennamen richtet sich die Genuszuweisung in Analogie zur Pronominalisierung nach der soziopragmatischen Unterscheidung. Auf Grund von sozialer oder affektiver Nähe weisen die Beispiele (12-16) neutralen

${ }^{7}$ Zur Verbreitung des haupttonigen, isolierten Personalpronomens ,es‘ im Schweizerdeutschen cf. SDS III: 200. 
Artikel, die Beispiele (17-19) neutrale Possessiv- und Demonstrativartikel auf. Übereinstimmung zwischen natürlichem Geschlecht und (neutralem) Genus ist dabei aufgehoben. ${ }^{8}$ Dabei ist hervorzuheben, dass Männerrufnamen, anders als im Wallis (cf. Christen 1998: 278; sowie unten Kapitel 2.2), nicht diminuiert sein müssen, um ins Neutrum gesetzt zu werden.

Beispiele mit Artikel und attributivem Adjektiv:

(12) $\underline{d s}$ sielig Edmond

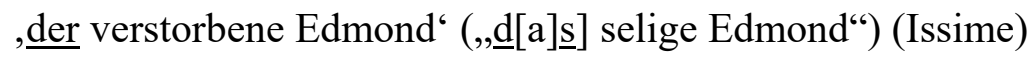

(13) $\underline{d s}$ ljib Jacobo ischt mein niizjing

, der liebe Jakob ist mein Neffe' (Issime)

(14) $\underline{d s}$ ellen Anna

, die arme Anna“ (Issime)

Ohne begleitendes Attribut (in Issime und Gressoney eher unüblich; gängig dagegen in Rimella und in Bosco Gurin): ${ }^{9}$

(15) ts Méje, ts Riko

,die Maria, der Rico“ (Rimella, Bauen 1978: 119f.)

(16) $\underline{\text { ts }}$ Roosi, $\underline{\text { ts }}$ Hansi

(Bosco Gurin, Gerstner-Hirzel 1979: 183)

Beispiele mit Possessiv- und Demonstrativartikel:

(17) $\underline{\text { meis }}$ Jacobo, meis Cecilia, meis Valentino

,mein Jacobo, meine Cecilia, mein Valentino‘ (Issime)

Neutrum-Kongruenz zwischen Prädikativum und Subjekt in den folgenden zwei Beispielen:

(18) ljiibs wi meis Claudio isch khe mensch on d weelt

, lieb wie mein Claudio ist kein Mensch auf der Welt‘ (Issime)

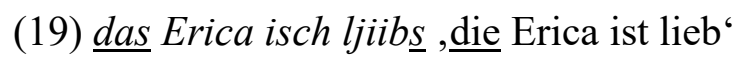

das Samuele isch ljiibs , der Samuel ist lieb“(Issime)

\subsection{Neutrum-Zuweisung: Rezente Ausweitung und Generalisierung}

Seit der zweiten Hälfte des 20. Jahrhunderts sind die Distanz- und Respektsverhältnisse in den kleinen dörflichen Walsergemeinschaften einem starken Wandel ausgesetzt. Geringe soziale Schichtung bei hohem gegenseitigem Bekanntheitsgrad führen dazu, dass sich die soziopragmatischen Bedingungen für die Genuszuweisung lockern. Lokale Hierarchien verlieren an Bedeutung; dies gilt auch für die intrafamiliären Hierarchien und Respektsverhältnisse. Der Brauch, Eltern und Grosseltern mit „Ihr“ anzusprechen, ist bei Kindern, geboren in der zweiten Hälfte des 20. Jahrhunderts, nicht mehr üblich.

\footnotetext{
${ }^{8}$ Beispiele mit variabler Genus-Zuschreibung sind in Kapitel 5.2 angeführt.

${ }^{9}$ Einzelnachweise für das Vorkommen des bestimmten Artikels vor männlichen Personennamen in den Südwalser Dialekten in SDS III: 141. - Cf. das Kapitel „Bestimmter Artikel vs. Artikellosigkeit bei Personennamen“ in Glaser (2008: 92-98), wo das Thema in Hinsicht auf areale Verbreitung im europäischen Raum behandelt wird. - Zum Artikelgebrauch vor Vornamen im Italienischen cf. Serianni (1989: 169).
} 
Darauf reagieren die Dialekte mit dem Rückzug der am Abstands- oder Respektsverhältnis orientierten Pronominalisierung. Die pronominale Referenz erfolgt zusehends geschlechtsindifferent im Zuge einer Generalisierung des Neutrums. Anders als in binnendeutschen und in (fast allen) schweizerdeutschen Dialekten bleibt, wie bereits festgehalten, die Neutrum-Zuweisung nicht auf weibliche Personen und -bezeichnungen beschränkt, sondern bezieht sich gleicherweise auf die männlichen (cf. bereits Bauen 1978: 117-119). Während bei der traditionellen Regelung die Neutralisierung bei Personen noch dem Prinzip der Nähe unterlag, gilt sie hier nun generell.

Ein Vergleich mit dem Wallis erweist sich als aufschlussreich (cf. Christen 1998: 276-279; Baumgartner/Christen 2017). Dort ist die Personen-Neutralisierung bei Männern ebenfalls belegt (cf. Wipf 1910: 141); Id. I: 512; SDS III: 200, Leg.; Bucheli Berger 2006: 96; cf. Karte 2: „Es (Paul) isch 71“ [ist 71 Jahre alt]), indes mit signifikanten Unterschieden, wie dies aus Christen (1998) hervorgeht. Während sich in den Südwalser Dialekten - und hier vor allem in Issime, Rimella (cf. Bauen 1978: 118) und Formazza (cf. Dal Negro 2004: 156) - die Neutralisierung verallgemeinert, schränkt sie sich im Wallis tendenziell ein. In dem älteren, von Wipf (1910) dokumentierten Sprachgebrauch wurde sowohl diminuierten wie nicht-modifizierten Vor- und Familiennamen von Männern neutrales Genus zugewiesen; seit dem Ende des 20. Jahrhunderts sind die nicht-modifizierten Namensformen davon auszunehmen, denn nun ziehen nur noch diminuierte Namen neutrales Genus nach sich. Diese morphosyntaktisch bedingte Restriktion ist in den Südwalser Dialekten unbekannt: Dem einschränkenden Gebrauch der Neutralisierung im Wallis entspricht in den Südorten ein ausgreifender.

Gänzlich verdrängt die neutrale Genus-Zuweisung die maskuline und feminine jedoch nicht; diese lebt bei männlichen und weiblichen Bezugsgrössen in einem Überschneidungs- und Übergangsbereich variierender Genuskonstellationen weiter (cf. Kapitel 5).

Die Tendenz zur Neutrum-Generalisierung ist indessen ausgeprägt, sowohl bei der exo- und endophorischen Pronominalisierung von Personen und Personenbezeichnungen als auch beim Setzen von präonymischen Artikelwörtern vor Personennamen (cf. Kapitel 2.1). Besonders ausgeprägt ist diese Tendenz bei den Enklitika; die dabei auftretende Verletzung der Kongruenz wird wohl deshalb nicht als störend empfunden, weil sich die Enklitika im Übergang zu genusindifferenten Verbflexiven befinden (cf. Kapitel 6).

\section{Sprachexterne und sprachinterne Stützung der Genus-Zuweisung}

\subsection{Stützung der Maskulin-Feminin-Zuweisung}

\subsubsection{Höflichkeitsformen}

Zwei Formen von Distanz-Organisation sind hier anzuführen: die Höflichkeitsform mit der IhrRelation einerseits, die Hinzunahme des Titels und die Anrede für Honoratioren andererseits. Die Pronominalisierung nach Maskulinum und Femininum, nach der Geschlechts-Genus-Korrespondenz, stützt sich indirekt auf diese Traditionen.

Wenn das Ihrzen heute nur noch selten angewendet wird, war es noch in der ersten Hälfte des 20. Jahrhunderts gang und gäbe. Vor allem entsprach es einer familiären Hierarchie. Die Kinder redeten die Eltern mit $I h r$ an, und diese Gewohnheit hat sich bei einem kleinen Teil der vor der 
Jahrhundertmitte geborenen Gressoneyer und Issimer bis in die Neunzigerjahre erhalten (cf. auch SDS III: 199 Leg.).

Noch deutlicher wirkte sich die Personennennung mit Titel bzw. die Anrede von Honoratioren mit Titelnennung zugunsten der geschlechtsunterscheidenden Pronominalisierung und Artikelsetzung aus; mit ihr verband sich eine soziale Platzanzeige.

Beispiele:

(20) der hieru oarzet

, der Herr Arzt‘ (hieru ist sowohl Titel wie Appellativ, vergleichbar mit it. signore, frz. monsieur) (Issime)

(21) hieru hoptma , $\underline{\text { Herr Hauptmann“ }}$

(Anrede und ebenfalls Bezeichnung (mit Artikel) für den Gemeindepräsidenten, sindaco) (Issime)

(22) $\underline{d}$ frou barooni

,die Frau Baron' (Ehefrau eines Gressoneyer Barons, frou wie hieru (20) Titel und Appellativ) (Gressoney)

Die Lexeme gottu/gotta (+Vorname) bezeichnen Pate und Patin; in der Verwendung als Höflichkeitstitel entsprechend it. padrino und madrina waren sie noch bis vor einem halben Jahrhundert geläufig. Heute sind sie zwar noch bekannt, aber gänzlich aus der Mode gekommen (es sei denn für scherzhaften Gebrauch):

(23) gottu Samuele, gottu Ettore

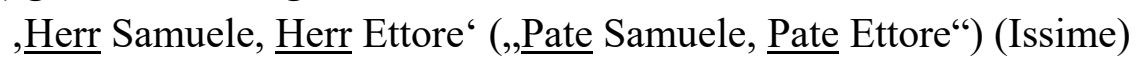

(24) schwig, olte narru, häd druf gsaid $\underline{d}$ gotta Maria, tua nis liebur vörbatu

,schweig, alter Narr, hat darauf die Frau Maria (,die Patin Maria“) gesagt, sag uns lieber Gebete auf‘ (Alagna, Giordani [1891] 1927: 102)

\subsubsection{Eine feminine Sonderform}

Im Pronomen-Inventar von Issime ist auf eine distanzmarkierende Sonderform hinzuweisen. Neben $d s c h i$, sie“ stellt sich in der 3. Person Singular Femininum die Subjekts-Vollform-Variante $d s c h o ̈ i$. Im Unterschied zu $d s c h i$ schränkt sich $d s c h o ̈ i$ ausschliesslich auf weibliche Personen ein, wogegen bei $d s c h i$ die Erstreckung auf Sachen nicht ausgeschlossen ist (ohnehin nicht bei enklitischem $=d s c h i$ ). $d s c h o ̈ i$ als spezifisches Feminin-Pronomen wird einerseits auf nahestehende Frauen bezogen, die hinsichtlich Alter oder Stellung in der Familie bzw. in der Gemeinschaft als Respektspersonen sozial ausgezeichnet sind, andererseits auf ferner stehende Frauen, denen man Achtung zuteil werden lässt, zu denen aber keine weiteren Beziehungen bestehen. Mit $d s c h o ̈ i$ setzt sich die soziopragmatische Ausrichtung fort, wie sie in Kapitel 2.1 dargelegt wurde, und es verstärkt sich gleichzeitig die Genus-Sexus-Koppelung. Mit diphthongiertem Vokal ist sie die stärker markierte Variante als dschi (cf. Zürrer 1999: 250-253). ${ }^{10}$

Beispiele:

\footnotetext{
${ }^{10}$ Cf. auch SDS III: 201, Id. VII: 12. In Gressoney ist nur $d s c h i$ üblich. - Zur Beziehbarkeit von $d s c h i$ auf Tiere und Inanimata cf. Kapitel 4.
} 
(25) dschöi het passurut alli de lebtag z eischeme

,sie (die Mutter der Gewährsperson) hat ihr ganzes Leben in Issime verbracht' (Issime)

(26) dschöi het toan de ménedsche un gchochut ds ganz zeit

,sie (die Mutter des Dorfpfarrers) hat während der ganzen Zeit (ihm) den Haushalt besorgt und gekocht' (Issime)

Mit Hilfe des femininen Wortbildungssuffixes - $u$ wurde $d s c h o ̈ i$ substantiviert zu $d s c h o ̈ i u$; in der Bedeutung, ältere, stattliche Frau', it. matrona, stellt sich dieses Wort zu den beiden anderen in Issime geläufigen Bezeichnungen, fümmela als unmarkierte und vrauwa als markierte Bezeichnung (für die fremde oder sozial höherstehende Frau). ${ }^{11}$

\subsection{Stützung der Neutrum-Zuweisung}

Zur Neutrum-Zuweisung, wie wir sie bei Personen, Personenbezeichnungen und Namen beobachten und in Kapitel 2.2 skizziert haben, können eine Reihe von Erscheinungen in Parallele gesetzt werden, wo ebenfalls das Neutrum bestimmend ist (cf. Kapitel 3.2.1 bis 3.2.5). Im Personenbereich spielt das dritte Genus offensichtlich eine Rolle, die ihm in den anderen alemannischen Dialekten nicht in gleichem Masse zukommt. Zu diesen Neutrum-,,lastigen“ Erscheinungen zählt das eigentlich neutrale, südwalserisch aber invariante Relativpronomen das sowie das Indefinitpronomen eswel- ,jemand', das gewohnheitsmässig ins Neutrum gesetzt wird; dazu gehören im Weiteren die unpersönliche Konstruktion für ,man', die Flexion des prädikativen Adjektivs und Partizips (mit prominentem Neutrum-Flexiv) und die mit dem Neutrum assoziierte Diminutivbildung.

\subsubsection{Relativpronomen}

Relativsätze werden in Issime und Rimella nicht mit wo eingeleitet (wie in Gressoney und generell im Schweizerdeutschen), sondern mit der Neutrumform des Relativpronomens, mit das. Da dieses das mit keinem anderen Genus alterniert, fungiert es nicht als Neutrum, sondern als invariante Relativpartikel. Mit ihr werden Personen ohne Rücksicht auf ihr Geschlecht pronominalisiert. In Rimella wird relatives das konkurrenziert von ke (<it. che).

Beispiele:

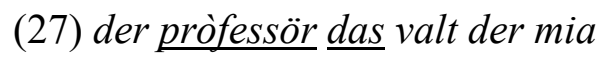

,[wer ist] der Lehrer, der dir am meisten (,mehr“) gefällt?‘ (Issime)

(28) rei ischt en kàrbinier das ischt z machu la multa

,hier ist ein Polizist, der im Begriff ist, eine Geldstrafe auszustellen`(Issime)

(29) der $\underline{\text { ma das }}$ isch=nu pchomut

,der Mann, der ihnen begegnet ist‘ (Rimella, Bauen 1978: 121)

\subsubsection{Indefinit-, Interrogativpronomen}

Die Indefinitpronomen eswels ,jemand', itlies ,jeder einzelne', nid äis ,niemand, keiner' und das Interrogativpronomen wels, welches` erscheinen in Rimella, Bauen (1978: 120) zufolge, grundsätzlich - und wohl seit alters - in der Neutrumform:

\footnotetext{
11 Zum Wortfeld „Frau“ in der Mundart von Gressoney cf. Zürrer (1975: 68-70). - Zu der in den Südwalser Mundarten nicht belegten, vergleichbaren Sonderform ihns cf. Klein/Nübling (2019).
} 
(30) eswels hed mer schaid

,jemand hat mir gesagt' (Rimella, Bauen 1978: 120)

(31) wels gäid üf da

,wer geht dort hinauf?' (Rimella, Bauen 1978: 120)

\subsubsection{Unpersönliche (Ersatz-)Konstruktion für ,man'}

Im Dialekt von Rimella gibt es für die unpersönliche Konstruktion mit ,man ‘ einen Ersatz:12 Er besteht in der Verwendung des Pronomens 3. Person Singular Neutrum, ,es'. Darauf aufmerksam macht Kolmer (2009: 233-236), mit Bezug auf Bauen (1978: 50, und passim). Nach Kolmer ist diese Konstruktion nicht kontaktbedingt, wie Bauen annimmt, sondern beruht auf Eigenentwicklung. ${ }^{13}$

(32) stäid= $\underline{\text { is }}$ üf ,man steht auf" (,,steht es auf"), mad es ,man mag, man kann“ (,mag es“") (Rimella, Bauen 1978: 50)

(33) es meste macho d schjöle titschu und wältschu

„man sollte deutsch und italienisch Schule halten“ („es müsste machen“) (Rimella, Bauen 1978: 142)

(34) d lit meschtet es grifu sül fat

,die Leute müsste man (,müsste es ${ }^{“}$ ) auf frischer Tat ertappen“ (Rimella, Bauen 1978: 142)

\subsubsection{Prädikative Adjektiv- und Partizipflexion}

Die Flexion des prädikativen Adjektivs und Partizips ist eine auffällige morphosyntaktische Besonderheit des Höchstalemannischen (Fleischer 2007; Zürrer 2009: 132f.; SDS III: 256258); sie führt dazu, dass die Kongruenzanzeigen hier zahlreicher sind als sonst in alemannischen Dialekten. Im Höchstalemannischen der Südwalser zeigen sich nochmals andere Verhältnisse. Die grassierende Zuteilung des Neutrums zu Personen und Personenbezeichnungen zieht hier folgerichtig eine Zunahme der neutrum-flektierten Prädikativa auf Kosten der femininen und maskulinen nach sich. Die neutrale Flexion des Prädikativs geht sogar noch einen Schritt weiter, indem wie in Beispiel (37) ein männlicher Personenname, dem ein maskuliner Artikel vorangestellt ist, neutral und nicht, wie erwartet, maskulin flektiert. ${ }^{14}$

Nachfolgend werden Beispiele für prädikative Adjektive (35, 36, 37, 39) und Partizipien (38, 40) im Neutrum angeführt.

Mit persönlichem Bezug auf männliche bzw. weibliche Personen und Personennamen:

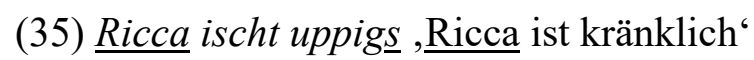

Peter ischt uppigs, Peter ist kränklich' (Issime)

(36) Claudio isch guts tun aldsch, isch guts z melje, isch guts z troan d milch

,Claudio ist gewandt in allen Dingen, melken, die Milch (in die Molkerei) tragen` (Issime)

\footnotetext{
$12 \mathrm{Zu}$,man“ im Schweizerdeutschen cf. SDS III: $229 \mathrm{f}$.

13 Die für Issime typische Konstruktion mit unpersönlichem Reflexivum als Alternative zur Verwendung von $m u$ ,man“ hat dagegen ihr Vorbild im si impersonale des Italienischen: schwetzt=dschi ,man spricht‘ (,,spricht sich“, cf. it. si parla), cf. Zürrer (1999: 312-316).

${ }^{14} \mathrm{Cf}$. auch die Inkongruenz im Beispiel (50): der wolf isch nit bljends ,der Wolf ist nicht blind‘.
} 


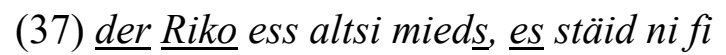

, der Rico ist immer müde, es geht ihm nicht gut' (it. non sta bene) (Rimella, Bauen 1978: 119)

Bei der Artikellosigkeit der Personennamen (cf. die Beispiele $(35,36))$ ist das neutrale Prädikativum (uppigs, guts ) das einzige Neutrum-Indiz.

Mit unpersönlichem Bezug und Indefinitpronomen als Bezugswort:

(38) isch als abandunuruts

,es ist alles verlassen“ (Issime)

Mit unpersönlichem Bezug auf ein Korrelat-es (39), ohne Bezugswort (40):

(39) $\underline{s}$ isch heps, z schii gschends

,es ist schön, gesund zu sein' (Rimella, Bauen 1978: 124)

(40) bhemt toans,, (es ist) sofort getan' (Issime)

Isoliert, als Nennform (Adjektive):

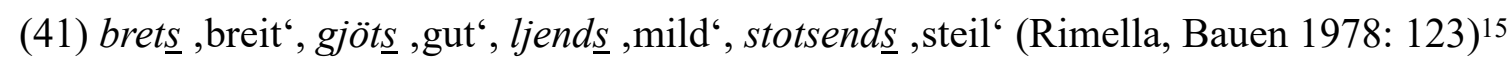

\subsubsection{Diminutive}

Die Diminutivbildung ist in den Südwalser Dialekten von hoher Produktivität und verzweigt sich in eine Palette von Neutrum auslösenden Suffixen:

(42) $-j i,-l i,-i,-(e l) t i,-s i, z i,-(t) s c h i{ }^{16}$

(cf. Cigni 2003: 59-74; Zürrer 1982: 96f.; Gerstner-Hirzel 1979: 183; SDS III: 155f.)

Einschränkend ist festzuhalten, dass die Diminuierung Appellativa betrifft; Namen sind davon weitgehend ausgeschlossen (cf. oben Kapitel 2.1 und gleich nachfolgend).

Eine Reihe von lexikalisierten Diminutiven ist besonders auffällig. Es handelt sich um Bezeichnungen für männliche und weibliche Personen, sowohl Kinder wie Erwachsene, die auf Grund ihres Suffixes neutrales Genus aufweisen: etterli, Junggeselle' $\left(<\right.$ ettru, Onkel' $\left.{ }^{\circ}\right)$ und muemi ,unverheiratete Frau' $(<$ muema ,Tante'), beide Bezeichnungen mit scherzhafter Konnotation, aber ohne usuelle Abwertung (Gressoney), analog etterllji und mumi (Issime); vrauli ,Fräulein‘, it. ,damigella‘ (Issime), bubji ,Junge‘ und töchterlji ,Mädchen“ (Issime), buffelti, buffji ,Junge“ und moati ,Mädchen“ (Gressoney), mandje ,Knabe, Bub, Sohn` und metje ,Mädchen` (Rimella, Bauen 1978: 119), gottelti ,Patenkind‘ (< gottu ,Pate‘, gotta ,Patin‘) (Gressoney).

Ein Vergleich mit den Verhältnissen im Wallis stellt auffällige Gegensätze heraus (cf. Christen 1998: 276-279). Personennamen, und zwar Vor- wie Familiennamen, werden im Wallis mit Vorliebe diminuiert, und entsprechend werden referierende Artikel und Pronomen mit relativ

\footnotetext{
15 „Zweifellos ist der vielfältige und häufige Gebrauch des Neutrums mit ein Grund dafür, dass die Rimeller auch für die Nennform der Adjektive das Neutrum vorziehen“ (Bauen 1978: 123.). Adjektive im Dialekt von Saley, ebenfalls in neutraler Nennform, sind angeführt in Frei (1970: 224f.).

16 Zu Diminutivbildungen in „Gebirgsmundarten“ cf. Szadrowsky (1930: 287f., hier auch zum Zusammenhang zwischen Diminution und stark anwachsender Neutralisierung), sodann im Schweizerdeutschen bzw. im alemannischen Raum, unter besonderer Berücksichtigung der Verhältnisse im Wallis, auch unter pragmatischen Aspekten, Christen (1998) sowie Baumgartner/Christen (2017: 132-137, mit Verbreitungskarten: Abbildung 1-4).
} 
hoher Frequenz ins Neutrum versetzt. Bei den Südwalsern ist Diminuierung von Vornamen selten, wenn nicht ausgeschlossen, weil hier italienische Rufnamen (Marco, Attilio, Andrea, Elide etc.) gebräuchlich sind, die nicht diminuiert werden. ${ }^{17}$ Ebensowenig haben die diminuierten, mit dem Neutrum kongruierenden Walliser Familiennamen in Gressoney und Issime eine Entsprechung. Im Wallis erfährt die eben festgehaltene Neutralisierung hinwiederum eine bemerkenswerte Einschränkung: Die normalen Namensformen von Männern werden anders als die diminuierten durchwegs mit dem Maskulinum assoziiert - eine Regel, die in keiner Weise für die Südwalser Mundarten gilt. Für Frauennamen besteht im Wallis variable Zuweisung: Feminines Genus kann sowohl bei der Normalform der Personennamen wie bei deren Diminutivform zugewiesen werden. Auch hierin weichen die Südwalser Verhältnisse ab: Hier gilt generalisierende Neutralisierung; Pronominalisierung im Femininum bildet die Ausnahme und ist abhängig von den in Kapitel 2.1 angegebenen soziopragmatischen Einschränkungen.

\section{Genus-Zuweisung in nicht-menschlichen Bereichen (Tiere, Objekte)}

Die Neutralisierung ist, wie wir gesehen haben (vor allem in Kapitel 2.2), im menschlichen Bereich dominant: Personen, Personenbezeichnungen (Appellative), Namen werden bei der Pronominalisierung und bei Artikelformen, einem starken Trend folgend, ins Neutrum gesetzt, ungeachtet, ob es sich dabei um weibliche oder männliche Personen und Personenbezeichnungen handelt. Eine analoge Neutralisierung ist im Bereich des Gegenständlichen nicht zu beobachten: Bei Pronominalisierung bleibt Genus-Übereinstimmung zwischen Referenz und Bezugswort intakt erhalten (cf. auch Dal Negro 2004: 156). Bei Tieren ist Neutrum-Zuweisung möglich, sofern zu ihnen ein durch Affekte gezeichnetes Verhältnis besteht (48-50), im anderen Fall gilt dieselbe Regel wie bei Objekten.

Der Gebrauch der Vollformen des Personalpronomens ist beim Bezug auf Sachbezeichnungen unüblich; geläufig ist die Referenz mit Demonstrativpronomen (cf. Wipf 1910: 141; Nübling 1992: 265f.), vor allem bei Emphase, sonst mit Klitika, sofern nicht elliptische Fügungen (ohne Subjektspronomen) vorgezogen werden. Die Beispiele (43-47, Tiere) und (51, 52, Inanimata) belegen, wie sich sowohl Klitika wie Demonstrativa ans Genus des Bezugsnomens anpassen (durchgehende grammatische Kongruenz), während die Beispiele (48-50) mit inkongruentem Neutrum davon abweichen (Haustier in $(48)$, wilde Tiere in $(49,50)$ ).

Beispiele:

Tiere:

(43) woa ischt dar hoanu? der ischt hinner ds gmach

,wo ist der Hahn? Der ist hinter dem Haus' (Issime)

(44) di ischt gganget an $i$ stell

,[wo ist die Ziege hingegangen?] Die ist an eine [gefährliche] Stelle gegangen“ (Gressoney)

\footnotetext{
17 Zur Modifikation von Eigennamen im Italienischen (ipocoristici, diminutivi) cf. Serianni (1989: 26 (nota), 549 551); die Diminuierung ist eingeschränkt, abhängig von Dialekten, sozialen Schichten, von den einzelnen Namen. Im Walserdeutschen sind die verbreiteten italienischen Vornamen in der Normalform gebräuchlich, bei deutschen Rufnamen (Fritz, Oskar, Heidi), die heute nicht mehr vergeben werden, ist Diminuierung ebenfalls unbekannt.
} 
(45) aber schi hed no gizaablu, dischi gemschu, schi ischt nid rächt gsii tuot $\underline{i}$ ,aber sie hat noch gezappelt, diese Gemse, sie war nicht richtig tot' (Macugnaga, Waibel 1985: 75)

(46) der hotschpal, er hed hupt en ds wasser ,der Frosch, er ist ins Wasser gesprungen“ (Rimella, Bauen 1978: 117)

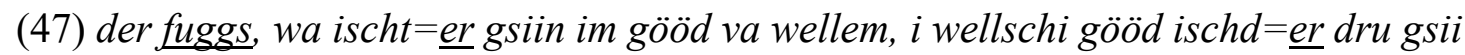
,der Fuchs, auf wessen Gut ist er gewesen, in wessen Gut war er drauf?' (Macugnaga, Waibel 1985: 92)

Mit Genuswechsel vom Substantiv zum referierenden Pronomen (Dialogausschnitt):

(48) [Mutter, die Katze tadelnd:] daasch proprio en tummi chatzu/[Tochter, verteidigend:] ferum hät $=\underline{s}$ geng de hunger ,das ist wirklich eine dumme Katze/[sie verhält sich so] weil sie immer Hunger hat (attributives Adjektiv mit F.-Endung [-i $]$ vs. Klitikon im Neutrum $[=\underline{\underline{s}}]$ ) (Gressoney, Zürrer 1999: 106)

Mit emotivem, auf wilde Tiere bezogenem Neutrum:

(49) der beero $[\ldots] \underline{\text { es }}$ ässt mer allu d gäis

,der Bär [...] er frisst mir alle Ziegen“ (Rimella, Bauen 1978: 119)

(50) der wolf isch nit bljends

,der Wolf ist nicht blind' (prädikatives Adjektiv mit N.-Endung), (Rimella, Bauen 1978: 119)

Inanimata:

(51) d karèma ischt a storhe wein, ischt töiri, wa gsuuchti. der ischt töiri ,der Wein von Carema ist ein schwerer Wein, (er) ist teuer, aber gesucht. Der ist teuer ${ }^{6}$ (abgelehnt: eer ischt töiri) (Issime)

(52) $i$, de rogge maan $=i$ niit, de han $=i$ ni geere

,ich mag den Roggen nicht, den habe ich nicht gern' (Gressoney)

\section{Innovative Lösungen: Zuweisung in wechselnden Zusammenhängen}

Wie die Genuszuweisung bei der Pronominalisierung im Südwalserdeutschen zwei Prinzipien folgt, wobei im einen Fall alle drei Genera funktionstragend sind, im anderen hingegen nur noch das Neutrum, ist in Kapitel 2 dargestellt worden; beide Prinzipien sind gegenwärtig wirksam, wenn auch die Neutralisierung stark überhandnimmt. Dabei lässt die ambige Situation verschiedene Lösungen zu, reduktive ebenso wie komplexe. Einerseits setzt sich die Neutralisierung penetrant durch; durch die Ausschaltung der maskulinen und femininen Pronomen-Zuweisung führt sie grammatische Inkongruenz herbei (Kapitel 5.1). Andererseits hält sich die Maskulin-Feminin-Zuweisung (Kapitel 5.2) und geht ausserdem neuartige Verbindungen ein (Kapitel 5.3 und 5.4). Ein Übergangsbereich variierender Genus-Zuweisungen ist dabei im Entstehen, ein Spielraum von Kongruenzen und Inkongruenzen grammatischer und pragmatischer Art, von Genus-Wechsel ,,ad hoc“. Diese Zuweisung erlaubt eine gewisse Wahl- und Kombinationsfreiheit und folgt - mit der gewichtigen Ausnahme von Kapitel 5.1 - noch keinem durchgängigen Automatismus, der die Genuswahl aufs einzige Neutrum einschränkt. 


\subsection{Einseitige Neutrum-Zuweisung}

Stellt sich das durch ein soziales Näheverhältnis motivierte neutrale Pronomen in unmittelbare Nähe zu seinem maskulinen oder femininen Bezugsnomen, kann sich eine besonders krasse Wirkung einstellen. Die Beispiele mit pronominalen Vollformen dürften dies augenfällig belegen. In $(53-57,61)$ führt der Anschluss des referierenden neutralen Pronomens ans feminine oder maskuline Bezugsnomen zu einem Genera-Clash, zu einer höchst auffälligen Diskordanz. Grammatische Kongruenz, die zwischen den Bezugsgrössen die Regel bildet, wird ausgesetzt. Inkongruenz als Folge ist auch bei den weniger krass wirkenden weiteren Beispielen festzustellen, so bei den Beispielen mit Klitika (58-60). In soziopragmatischer Hinsicht stellt sich dagegen Übereinstimmung ein: Geschwister, Sohn und Freund sind der pragmatischen Gepflogenheit gemäss ins (konforme) Neutrum gesetzt.

Die auffällige Erscheinung, die sich trotz grammatischer Inkongruenz ausbreitet, lässt sich allerdings auch anders erklären: nicht über die soziopragmatische Rolle des Neutrums, womit das Nähe-Verhältnis gemeint ist, sondern über die radikale Verallgemeinerung des Neutrums im Pronominalbereich. Das Neutrum ist im Begriff, sich als Genus für Personen schlechthin (cf. Kapitel 1.1) zu etablieren; seine Setzung bedarf keiner exophorischen Motivation. Die beiden Erklärungen schliessen sich nicht aus: Der allgemeine Trend zum neutralen Genus für Personen vermindert die (störende) Inkongruenz, und das in der Gemeinschaft sich ausbreitende soziale Näheverhältnis erleichtert die Versetzung von Personen ins pronominale Neutrum.

(53) wer isch parturut? iis, mein bruuder

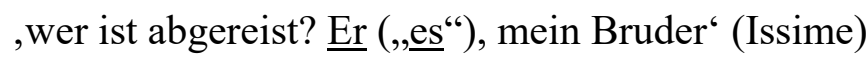

(54) wer isch gsing operé im spital? iis, mein wätta

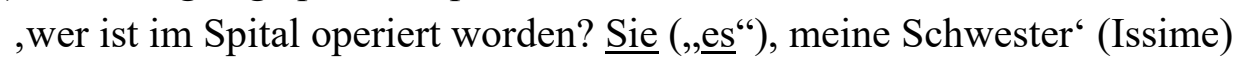

(55) wer het gwunnet d gaaaru? ääs, min $\underline{\text { bueb }}$

,wer hat den Wettlauf gewonnen? Er („es “), mein Sohn“ (Gressoney)

(56) wels ischt gganget? d wääta ol de brueder? isch ääs gganget, de $\underline{\text { brueder }}$

,wer (,welches“) ist gegangen? Die Schwester oder der Bruder? $\underline{\operatorname{Er}}$ (,"吕“) ist gegangen, der Bruder $^{6}$ (Gressoney)

(57) äi meischteri $[\ldots]$ wa hät alls befole $\underline{\ddot{a} s}$ hät alls welle $\underline{a ̈ s}$ machu $\underline{\ddot{a} s}$

,eine Hausherrin [...] die alles herumkommandiert hat, $\underline{\text { sie }}$ (,, $\left.\underline{e s}^{\text {“c }}\right)$ hat alles machen wollen, sie (Formazza, Dal Negro 2004: 153)

Beispiele mit Klitika:

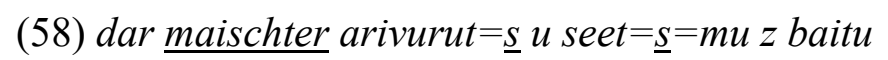

,der Besitzer kommt herbei und sagt ihm (bittet ihn) zu warten“ (,der Meister es kommt und es sagt ihm zu warten“) (Issime)

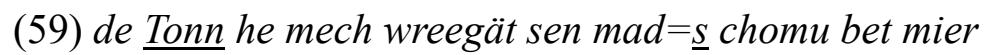

,der Anton hat mich gefragt, ob er (,,[e] $\underline{s}^{\text {““) }}$ mit mir kommen könne“ (Rimella, Bauen 1978: 118)

(60) diö schiwer gschi da bei d ajo wa hed $=\underline{s}$ gwäscht

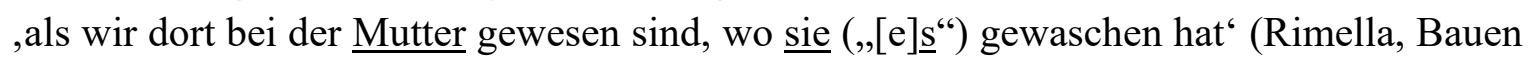
1978: 119) 
Im folgenden Beispiel (61) nimmt die betonte Neutrum-Vollform, iis, das vorausgegangene feminine Nomen, wätta ,Schwester', auf und schliesst mit proklitischem Subjektspronomen, $s=$, im selben neutralen Genus direkt an iis an:

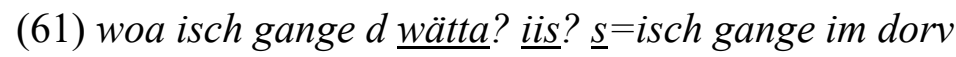

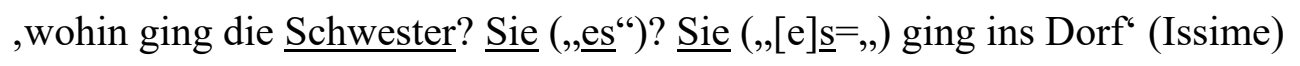

\subsection{Mehrseitig motivierte Maskulin-/Feminin-Zuweisung}

Die Maskulin-/Feminin-Zuweisung vermag sich zu behaupten, nicht zuletzt dank komplexerer Motivation. Die markierte Genuswahl muss sich nicht notwendig auf das in Kapitel 2.1 genannte Distanz- oder Respektsverhältnis beschränken, das zwischen Sprecher oder Sprecherin zur Bezugsperson besteht. Zusätzliche Variable können die Wahl des maskulinen oder femininen Artikels mitbestimmen und die Regelung gegenüber der nivellierenden Neutrum-Wahl aufrecht erhalten. Darauf aufmerksam geworden bin ich in einer Unterhaltung mit Elide, einer meiner Gressoneyer Gewährspersonen, Lehrerin in ihrer Heimatgemeinde, mit der ich gerne Fragen zu ihrem Dialekt diskutierte. Ausgangspunkt der Erörterung war der präonymisch gesetzte Artikel zu weiblichen und männlichen Rufnamen. Auf die supponierte Frage nach dem Ergehen von Angehörigen, im gegebenen Fall von Elides kranker Mutter und von ihrem (damals kranken) Ehemann, erwogen wir die folgenden Antwortvarianten (62, 63 vs. 64, 65):

(62) ds ellen Emma, weisch witte isch $=\underline{s}$ chrang (sagt die Tochter in Bezug auf ihre Mutter) ,die arme Emma, weisst du, wie (sehr) sie krank ist“ (,das arme Emma, weisst du wie ist es $\mathrm{krank}^{6}$ ) (Gressoney)

(63) $\underline{d s}$ ellen Ettore, weisch witte isch $=\underline{s}$ chrang (sagt die Frau in Bezug auf ihren Mann) ,der arme Ettore, weisst du, wie (sehr) er krank ist“ (,das arme Ettore, weisst du wie ist es $\mathrm{krank}^{6}$ ) (Gressoney)

Mit dem neutralen Artikel, $d s$, und dem gleichfalls neutralen, enklitischen Referenzpronomen, $=S$, ist eine Wahl getroffen, die unmarkiert erfolgt und das Näheverhältnis zwar mitsetzt, aber nicht eigens signalisiert.

Anders dagegen in den folgenden Varianten $(64,65)$, wo Artikel und Pronomen sowie das prädikative Adjektiv durch geschlechtsspezifische Formen Markierung anzeigen:

(64) $\underline{d}$ ellen Emma, dschi ischt chrangi

, die arme Emma, sie ist krank' (Gressoney)

(65) der ellen Ettore, er ischt chrange

, der arme Ettore, er ist krank' (Gressoney)

Mit der Wahl des femininen oder maskulinen Artikels drückt sich erwartungsgemäss einmal mehr soziale Distanz aus; nur richtet sie sich nicht auf die Bezugsperson - Mutter oder Ehemann - aus, sondern auf die sich erkundigende supponierte (Dritt-)Person, der gegenüber sich die Sprecherin, Elide, zurückhaltend gibt, indem sie ihre Betroffenheit für sich behält. Mit der distanzmarkierenden Genuswahl wird hier ein variables, mehrseitiges Personenverhältnis anvisiert.

In grammatikalischer Hinsicht stellt sich - im Gegensatz zu nähebestimmter $(62,63)$ oder pauschal nivellierender Neutralisierung wie in Kapitel 5.1 - bei $(64,65)$ keine Inkongruenz ein. 


\subsection{Divergierende Zuweisung}

Divergenz sind wir bereits im letzten Kapitel begegnet, nur wurde sie nicht thematisiert. Dort fielen die Antworten auf die selbe Frage - die Frage nach dem gesundheitlichen Befinden bestimmter Personen - divergierend aus, und zwar insofern als auf unterschiedliche Personenbeziehungen mit differierendem Genus reagiert wurde (neutrales in $(62,63)$ vs. feminines/maskulines in $(64,65))$. Auch im folgenden Beispiel (66) lässt sich divergierende Genuszuschreibung erkennen, nur verteilt sie sich nicht auf zwei Zugriffe pro Person, sondern auf einen einzigen: Im selben Satz verschränken sich die divergierenden Genera. Die Äusserung findet sich in einer in Issime gemachten Tonaufnahme aus den Neunzigerjahren: Einer betagten Bekannten wird ein guter Gesundheitszustand attestiert.

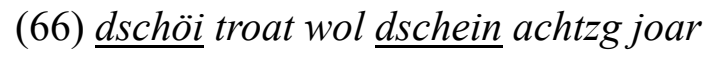

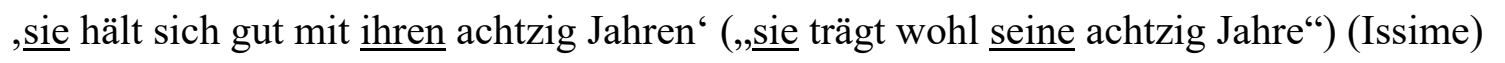

Die Referenz besteht hier nicht zwischen einem Nomen und einem darauf (anaphorisch oder kataphorisch) Bezug nehmenden Pronomen, sondern aus zwei Pronomen, die in referierender Beziehung stehen: Aus einem (vollformigen) Subjektspronomen, dschöi, und einem Possessivpronomen bzw. -artikel, dschein. Mit der referierenden Funktion verbindet sich ein doppelter

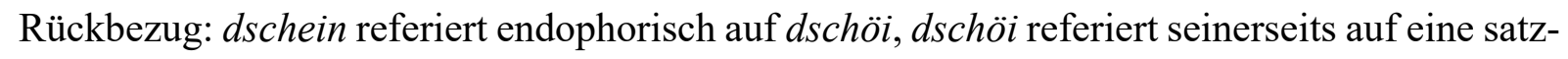
intern nicht angeführte, exophorische Bezugsperson; es ergibt sich eine koreferente Verkettung

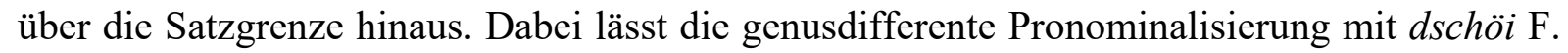
und $d s c h e i n$ N. grammatische Inkongruenz entstehen. In Anbetracht der achtzigjährigen Referenzperson wird mit dem femininen $d s c h o ̈ i$ die pragmatische Kongruenzregel zunächst eingehalten - hohes Alter präjudiziert Distanz bzw. Respekt und also (im Rahmen dieses Gesprächs) Feminin-Zuweisung -, mit dem nachfolgenden Neutrum des Possessivartikels, dschein ,sein“ (statt iira, ihr'), aber wird die soziopragmatische Markierung wieder aufgehoben. Die Neutralisierung dürfte wie bei den Beispielen in Kapitel 5.2 durch soziale Nähe motiviert sein, wenn nicht durch den unspezifisch generalisierenden Charakter des dritten Genus.

Die Genusneutralisierung durch $d s c h e i n$ ist vielleicht durch eine zufällige und nur vage Übereinstimmung mit den Kontaktsprachen zusätzlich motiviert. Man vergleiche z. B. frz. il/elle porte bien son âge, it. (egli/ella) porta bene la sua età. Es dürfte nicht ausgeschlossen sein, dass der für männliche wie weibliche Bezugspersonen einheitliche Possessivartikel, frz. son, it. sua, die issimedeutsche Form dschein suggerierte (und feminines iira ausschaltete). Nur: Da mit dschein eine neutrale Form (eigentlich eine maskulin-neutrale Einheitsform) das erwartete Femininum ersetzt, lässt sich die Parallele mit den Kontaktsprachen, die über kein drittes Genus verfügen, nicht schlüssig stützen (cf. Zürrer 1999: 249f.).

Im Unterschied zu den Beispielen in Kapitel 5.1, wo die Folge von Nomen und referierendem (neutralen) Pronomen ein wiederkehrendes Muster erkennen liess, handelt es sich bei der hier vorliegenden Folge von zwei im Genus differierenden Pronomen mit endo- und exophorischem Bezug um einen Einzelfall.

Ein weiteres Beispiel, ebenfalls aus einer Issimer Tonaufnahme (von 1990) dokumentiert einen Wechsel von Genus-Zuweisung im selben Satz. Auch hier ist der Wechsel unerwartet. Im Gegensatz zum vorangegangenen Beispiel (66) liegt nun aber nicht eine divergierende Genus- 
Zuweisung bei derselben Bezugsperson vor, sondern bei zwei in engste Parallele gesetzten Bezugspersonen.

Zur Situation: Der Issimer Marco, geb. 1968, berichtet mir von der Geburt seines ersten Sohnes und bezieht sich auf die damit verbundene genealogische Veränderung: Die Grosseltern werden Urgrosseltern. In seiner Äusserung bezieht er sich auf seinen eigenen Grossvater (väterlicherseits) und auf die Grossmutter seiner Ehefrau. Marcos Familie ist seit Generationen in Issime ansässig, die Familie seiner Ehefrau stammt dagegen aus dem Piemont und spricht nicht Walserdeutsch. Dieser Unterschied spiegelt sich nun, auf sprachlicher Ebene, in differierender Pronomensetzung. Indem Marco in einem ersten Schritt seinen Grossvater in Relation zum Neugeborenen setzt und in einem zweiten Schritt die Grossmutter seiner Ehefrau ebenfalls mit dem Baby in Verbindung bringt, verfährt er differenzierend: Den Grossvater setzt er ins Neutrum, die ,fremde“ Grossmutter ins Femininum. (Zu bisnonnu ,Urgrossvater" und bisnonne ,Urgrossmutter' cf. it. bisnonno, bisnonna.)

(67) ja, isch gchieme bisnonnu, auch $\underline{i \text { is }}$, und auch $\underline{\mathrm{d}}$ oaltu nonne isch auch gchieme bisnonne, auch $\underline{\text { dschöi }}$

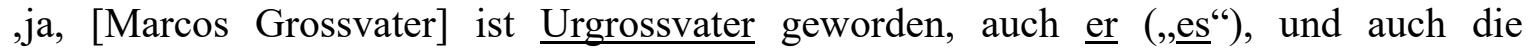
Grossmutter [seiner Ehefrau] ist $\underline{\text { Urgrossmutter geworden, auch sie' }}$ (Issime)

Die unterschiedliche Genuszuweisung ist auffällig. Man würde entweder Maskulin-/FemininPronominalisierung erwarten (eer/dschöi), wozu das Alter der Referenzpersonen Anlass gäbe, im anderen Fall aber, bei Abstraktion vom sozialen Faktor des Alters und unter Berücksichtigung des Näheverhältnisses des Sprechers zu den beiden Referenzpersonen, deren gemeinsame Versetzung ins Neutrum (iis). Genauer betrachtet bestehen nun aber subtile familiär-verwandtschaftliche Unterschiede in Marcos Beziehung und Einstellung zu den beiden Referenzpersonen; und diese bilden sich ab in der differierenden sprachlichen Umsetzung. Zum Grossvater (väterlicherseits) besteht von Kindesbeinen an familiär-vertraute Nähe, ausserdem verbindet ihn starke Empathie mit dem Mann, der seine Ehefrau verloren hat, die nun nicht die Freude erlebt, Urgrossmutter zu werden. Zur Schwiegermutter und besonders zu deren Mutter, d oaltu nonne, stellt sich das Verhältnis anders dar, denn trotz der Verwandtschaft durch Heirat besteht zu ihnen ein gewisser Abstand, motiviert durch Fehlen einer gemeinsamen Herkunft, einer gemeinsamen Familiensprache und gemeinsam verbrachter Jahre.

Das Beispiel zeigt, wie sich subtile Unterschiede im Empfinden in Genusunterschieden niederschlagen. Anders gesagt: In engster Abfolge bildet hier grammatische Variation empathische Differenz ab. Die anaphorischen Pronomen vermitteln durch die Annahme divergierender Genera unterschiedliche emotionale Wertungen, affektive Haltungen und Einstellungen gegenüber den beiden Referenzpersonen.

\subsection{Spontane Zuweisung}

Die nachfolgenden beiden Auszüge $(68,69)$ stammen aus einem 1995 aufgezeichneten Gespräch, bei dem ich mit Maria, einer 58-jährigen Issimerin, eine Bildergeschichte betrachtete und sie in einem Zwiegespräch die dargestellte Handlung in ihrem Dialekt wiedergeben liess ${ }^{18}$

\footnotetext{
18 Die Bilderfolge ist einem Zürcher Sprachlehrmittel entnommen und ibd. reproduziert (Zürrer 1999: 467).
} 
(cf. Zürrer 1999: 109-120); ich selber intervenierte mit Fragen zum Inhalt und zur dialektalen Ausdrucksweise, entweder auf Italienisch oder ebenfalls im Dialekt. Die Bilderfolge zeigt in satirisch-komischer Weise, wie ein gewiefter Arzt sich um eine Verkehrsbusse drückt, die ihm ein Polizist auferlegen möchte, indem er den Gesetzeswächter zum Patienten macht und krank schreibt, sich selber aber heimlich davonmacht.

Marias Wiedergabe interessiert uns in Hinsicht auf die Verweisformen für die beiden Protagonisten, den Polizisten und den Arzt. Auffällig ist gleich zu Beginn der Erzählung (68), wie Maria das Genus der auf den Arzt referierenden (enklitischen) Subjektspronomen ändert. Man

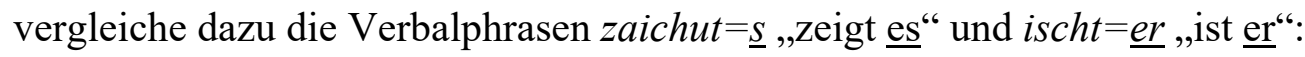

(68) $u$ hei zaichut $=\underline{\underline{s}}$ ds buursi, das ischt= $\underline{\text { er oarzat }}$

,und hier zeigt $\underline{\operatorname{er}}\left(,,=[\mathrm{e}] \underline{\mathrm{s}}^{\text {“ }}\right)$ auf die Arzttasche, (um zu verstehen zu geben,) dass er $\underline{\text { Arzt }}$ ist $t^{\star}$ (Issime)

Mit $i s c h t=\underline{\text { er oarzat }}$ stellt die Sprecherin Pronomen-Nomen-Kongruenz her, die sie aber gleich wieder aufhebt, indem sie zum Neutrum zurückfindet; erst in der dritten nachfolgenden Replik (69) gibt sie es zugunsten von zwei aufeinander folgenden maskulinen Enklitika ab (lait= $\underline{e r}$ ,legt $\underline{\text { er }}$ " und $t u t=\underline{e r}$, ,tut $\left.\underline{e r}^{(6)}\right)$

(69) hi na lait=erd han ouvn mun $u$ hi tut $=\underline{e r}$ schreibe

,hier legt er (dem Polizisten) die Hand auf den Mund und hier schreibt er [den Strafzettel]` (Issime)

Dieser letzte Wechsel dürfte sich aus der unmittelbar vorangegangenen Replik erklären, in der ich selber Dialekt sprach und den Arzt pronominal ins Maskulinum versetzte, was Maria veranlasste, sich anzupassen und meine Pronomenwahl für einen kurzen Moment zu übernehmen. Anschliessend blieb sie bis zum Schluss bei der Neutralisierung der auf den Arzt und den Polizisten bezogenen Pronomen.

Von insgesamt 28 von Maria für die beiden männlichen Protagonisten verwendeten Pronominalformen fallen 25 auf Neutra und nur 3 auf Maskulina (es sind die in den Auszügen (68) und (69) enthaltenen).

Die Beispiele $(68,69)$ legen zwar, herausgelöst aus dem Kontext, nahe, in der Genuszuweisung nichts Fixes zu sehen, keinen Automatismus; sie ist nicht zum Vornherein ausgehandelt, vielmehr vollzieht sie sich während der Verbalisierung, im Reden - es ist Genuszuweisung ,ad hoc“. Auf die ganze Erzählung bezogen, lässt aber Marias auffällige Häufung des NeutrumEnklitikons darauf schliessen, dass sich enklitisches $=s$ im Übergang befindet zu einer neuen Funktion (cf. Kapitel 6).

\section{Zuweisung und Reanalyse}

Die Bilderfolge mit Polizist und Arzt legte ich auch einer damals, 1995, 14-jährigen Issimerin, Francesca, vor. Sie entledigte sich der gestellten Aufgabe, die Bilderserie in eine fortlaufende Geschichte umzuwandeln, mit grosser Leichtigkeit, kannte sie doch solche Bilderfolgen aus dem Unterricht und ist sie ausserdem aufgewachsen mit Comics (it. fumetti); auch scheute sie sich nicht vor häufigem Code-switching. Hinsichtlich der Pronominalisierungen ist das Ergebnis eindeutig: Sie versetzt die beiden Protagonisten (der maischter alias der oarzat ,Arzt ${ }^{6}$, der kàrbinier ,Polizist', < it. ,carabiniere') durchwegs ins Neutrum. 
Beispiele aus der Wiedergabe:

(70) rei ischt en kàrbinier das ischt z machu la multa [...], wi is hät $\underline{\text { = }}=$ mu grad gschruube la multa [...], dar maischter vo la macchina arivurut $=\underline{\text { s }}$ de kursa u seet $=\underline{s}=$ mu z baitu z nöi machu la multa worum ischt iis ischt en oarzat un isch siin z machu una visita. [...] de iis giet $=\underline{s}$ la multa, giet $=\underline{\underline{S}}=m u$ il polso, $u n$ tut $=\underline{\underline{S}}$ lungu de relletsche, $t u t=\underline{s}=m u$ contare le pulsazioni, un dizuu tut $=\underline{S}=m u$ auh misurare $i$ battiti va il cuore, un tut $=\underline{S}=m u$ lungu di zungu [...] dar kàrbinier giet $=s$ in d han sta ricetta un dar oarzat loat $=\underline{s}=$ mu doa la multa, giet $=\underline{s}$ la macchina und gait $=\underline{S}$

,hier ist ein Polizist, der im Begriffe ist, eine Busse auszuteilen [...], wie er ihm gerade den Strafzettel geschrieben hat [...], eilt der Besitzer des Autos herbei und sagt ihm [bittet ihn] zu warten, ihn nicht mit einer Busse zu belegen, weil er Arzt ist und von einem Hausbesuch kommt. [...] Dann nimmt er [der Arzt] den Strafzettel, er misst ihm [dem Polizisten] den Puls und schaut auf die Uhr, er zählt die Pulsschläge, und dazu misst er ihm auch die Herzschläge und schaut seine Zunge an [und schreibt für ihn ein Rezept...], der Polizist nimmt dieses Rezept an sich (,,in die Hand“), und der Arzt lässt den Strafzettel zurück, er nimmt den Wagen und er geht [er macht sich aus dem Staub]‘ (Issime)

Von insgesamt 72 flektierten Verbformen (3. Person Singular) entfallen 43 auf die Variante mit enklitischem $=s, 29$ auf die enkliselose Form (vor allem ischt, ist ${ }^{`}$ ), keine einzige auf die Variante mit $=e r$.

Eine derart frequente neutrale Genuszuweisung bei persönlichen Pronomen mag nun doch Erstaunen hervorrufen, denn damit wird nicht nur die grammatische Kongruenz aufgehoben (cf. der oarzat $\mathrm{m}$. - iis $\mathrm{n} .-{ }_{s} \mathrm{n}$.), es wird ausserdem die erwartete (soziopragmatisch motivierte) Maskulin-Zuweisung den beiden zu respektierenden Protagonisten entzogen, und ohnehin wird die Kongruenz zwischen dem natürlichen und dem grammatischen Geschlecht fallen gelassen.

Noch grösseres Erstaunen dürfte der Bericht hervorrufen, den ein alteingesessener Issimer mittleren Alters, für den der Dialekt Alltagssprache ist, in Gegenwart von gleichaltrigen Issimer Nachbarn, ebenfalls bestens ausgewiesenen Mundartsprechern und -sprecherinnen, erstattete (71). Er berichtet von einer Konsultation mit seinem Sohn bei einem Arzt in Turin. Dabei beobachtet man durchgängiges Versetzen des Arztes, der dem Issimer bei der wiedergegebenen ersten Konsultation nicht bekannt war, ins Neutrum, und damit hört es sich an, als handelte es sich bei dem Arzt, entsprechend dem traditionellen Zuteilungs-Prinzip, um eine Person im symmetrischen Nähe-Verhältnis, um einen vertrauten Dorfgenossen (und nicht um einen unbekannten Turiner). Auch vom hierarchisch-asymmetrischen Verhältnis zwischen Arzt und Patient (oder - im vorliegenden Fall - Vater des Patienten) wird abstrahiert.

(71) si=wer gganget en oarzet $z$ türin doa. diischen oarzet het $=s \underline{\text { iis }}$ gviisuturut, do het $=\underline{s}$ gsait [...] ah, es het gsait $[\ldots]$

,sind wir zu einem Arzt dort in Turin gegangen. Dieser Arzt hat es [das Kind] untersucht,

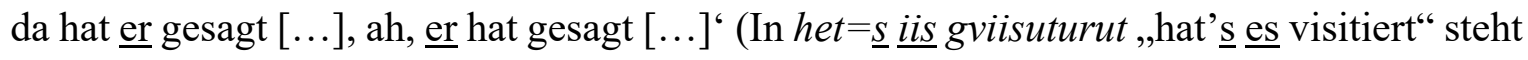
enklitisches $=\underline{s}$ für den Arzt, die betonte Subjekts-Vollform $\underline{i \underline{i s}}$, es ‘ dagegen für das Kind) (Issime)

Die inflationär wirkende Verwendung der Verbformen auf $=s$ wirft erneut die Frage nach deren Status auf (cf. Kapitel 5.4). Kann man darin noch ein Subjektspronomen mit neutralem Genus 
erkennen? Stellt man die annähernd völlige Verdrängung der maskulinen und femininen Klitika durch das generalisierte Neutrum-Klitikon in Rechnung, drängt sich eine Neubestimmung auf. Indem die Enklise einer Reanalyse unterzogen wird, verliert das $=s$-Enklitikon seine relative Eigenständigkeit, es büsst die Genusanzeige und damit die referentielle Rolle ein. Die pronominale Subjektsfunktion fällt dahin, das Klitikon ist syntaktisch funktionslos geworden. In einem Grammatikalisierungsprozess geht es etappenweise in die Verbalflexion über, zunächst noch als Verbalendung 3. Person Singular mit Genusmarkierung. Durch die Verdrängung der konkurrierenden maskulinen und femininen Klitika reduziert sich das dreigliedrige auf ein eingliedriges System und hebt sich damit auf. Das nunmehr genusbefreite $=s$-Klitikon mutiert zum Flexiv und verstärkt durch den Anschluss an die bereits vorhandene Verbalendung deren morphologisches Profil (cf. Zürrer 1999: 316-377; Dal Negro 2004: 170-180). ${ }^{19}$

Kontaktsprachlicher Einfluss dürfte hier mitbeteiligt sein. Subjektslos konjugierte Verbformen bilden im Italienischen den Normalfall; im Walserdeutschen wird durch den Übergang enklitischer Verbformen in elliptische eine Anlehnung an die Kontaktsprache manifest; die Erscheinung erfasst über die hier behandelte 3. Person Singular hinaus alle Paradigmapositionen. Es handelt sich freilich auch dabei um ein Übergangsphänomen, das durch andere Bildungsmuster konkurrenziert wird (Beispiele in Zürrer 1999: 367f.).

\section{Sprachgeschichtlicher Rückblick}

Die Neutrum-Zuweisung bei der Pronominalisierung männlicher und weiblicher Personen und ihrer Bezeichnungen ist nicht neusten Datums. Sie hat aber, trotz andauerndem Fortbestehen soziopragmatischer M.F.N.-Zuweisung, in den letzten Jahrzehnten des 20. Jahrhunderts überhandgenommen und sich - in Issime und Rimella vor allem - verallgemeinert. Sie lässt sich aber schon in schriftlichen Dialektaufzeichnungen aus dem Anfang und der Mitte des 19. Jahrhunderts spurenweise nachweisen. Die Belege finden sich in den Übersetzungen der Parabel vom Verlorenen Sohn, die in zwei Quellen überliefert sind. Bei den frühesten Zeugnissen (von 1807 und 1810) handelt es sich um Aufnahmen aus der Zeit des Napoleonischen Kaiserreichs, veranlasst vom Innenministerium unter der Leitung von Baron Coquebert de Montbret ${ }^{20}$. In der Nachfolge Coqueberts hat der Württemberger Germanist Albert Schott seinerseits das Gleichnis übersetzen lassen und die Aufnahmen eigenhändig transkribiert; sie sind enthalten in Schott (1842). Aus diesen frühesten Walser Dialekttexten führe ich Neutralisierungen an, die auf den Parabel-Protagonisten, den jüngeren, den ,verlorenen“ Sohn, rekurrieren (72, 73, 75, 76); von den beiden anderen Personen wird der ältere, beim Vater verbliebene Sohn einzig in der Issimer Version von Schott ins Neutrum gesetzt (74). In keiner Version erfolgt Neutrum-Pronominalisierung des Vaters. ${ }^{21}$

\footnotetext{
${ }^{19}$ Zu Pronominalklitika im Cimbro, einem südbairischen Dialekt, cf. Kolmer (2012: 159-222), zu einem Vergleich mit walserdeutschen Dialekten ibd. (197-202).

${ }^{20} \mathrm{Zu}$ Coquebert de Montbret cf. Ködel (2014). Die Südwalser Gleichnis-Übersetzungen sind gesammelt, transkribiert und kommentiert in Keller (1975 [1807]), eine erweiterte Neu-Edition ist in Vorbereitung, cf. Zürrer (2017). ${ }^{21}$ In den Übersetzungen in die Walliser und bündnerdeutschen Dialekte bei Stalder (1819) bleibt Neutrum-Zuteilung generell aus.
} 
(72) dernah, der jun 'e Sohn hăt als g 'leit z'sămme, und ischt g'gange in es witts Land, und da hăt' $\underline{\text { s alls aufg'gesse }}$

,danach hat der junge Sohn alles zusammengelegt und ist in ein entferntes Land gegangen, und da hat er alles aufgebraucht (,aufgegessen“)‘ (Issime, Keller 1975 [1807]: 133)

(73) deswegen hie dein Bruder ischt g'sen g'todt und ischt erlebet, $\underline{\text { is }}$ ischt g'sen Verlohrentz und $\underline{\text { is }}$ ischt g'funnent' $\underline{s}$

,weil hier dein Bruder tot gewesen ist und lebendig geworden ist, er ist verloren gewesen und er ist gefunden' (Issime, Keller 1975 [1807]: 134)

(74) der elter $\underline{\text { brûder }}$ isch nigg'sing z'frido, $\underline{\text { is }}$ hènnid uèlje ingjer chjèmme [...]. $\underline{\text { is }}$ hèd andchjède dschîm atto

,der ältere Bruder ist nicht zufrieden gewesen, er hat nicht herein kommen wollen [...]. Er hat seinem Vater geantwortet' (Issime, Schott 1842: 140)

(75) diz dî ŝu: is hèd g'gèsse alls dschîs gûd

,dieser dein $\underline{\text { Sohn, }}$ er hat sein ganzes Gut aufgezehrt' (Issime, Schott 1842: 140)

(76) uorum dass dîn brûder isch g'sing tüed unn 's-ischt irlèbed; $\underline{\text { is }}$ isch g'sing verlorens unn isch g'funnens

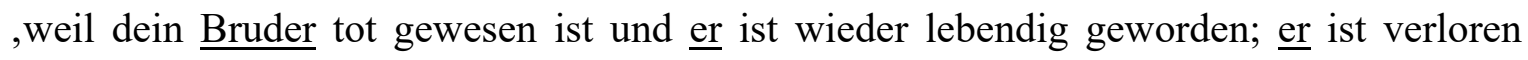
gewesen und ist gefunden' (Issime, Schott 1842: 140)

Alle fünf Belege in (72-76) nehmen die männlichen Personenbezeichnungen (,Bruder ${ }^{\star}$, ,Sohn“, brûder, sî) mit dem Neutrum-Referenzpronomen auf (is) bzw. mit enklitisch/proklitisch an die Verbform angefügtem - $s$, $s$-; ebenso enden die prädikativen Partizipien in $(73,76,77)$ auf neutralem $-s$.

Analog in der Fassung von Rimella:

(77) desche dîn brioder isch g'schîd tüed und isch erlèbed; es isch g'schîd verlüres un nuew hèwwers g'vunnod

,dieser dein Bruder ist tot gewesen und ist wieder lebendig geworden; $\underline{\text { er }}$ ist verloren

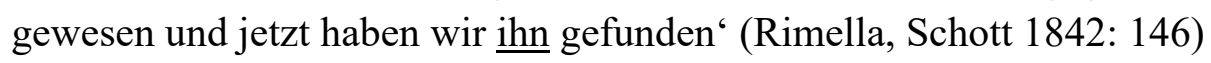

Ein späterer Beleg für Neutralisierung stammt aus einer Tonaufnahme von 1929 (dieselbe hat nicht die Parabel zum Gegenstand, sondern eine lustige Geschichte):

(78) aaber dets moal [...] ischt de wirgglig nit dä schuemachär gsii, $\underline{\text { ääs }}$ isch gäng hiä memmär gsii

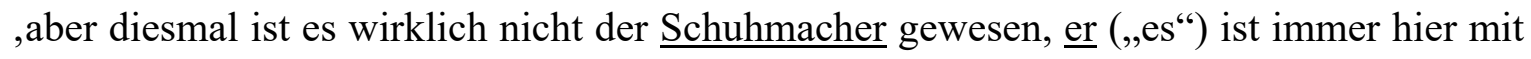
mir gewesen“ (Gressoney, Gysling/Hotzenköcherle 1952: 7)

\section{Fazit}

- Die Genus-Zuweisung bei der Pronominalisierung von Personen unterliegt in der zweiten Hälfte des 20. Jahrhunderts einem Wandel. Sie zeichnet sich durch den Vormarsch des Neutrums aus: durch Frequenzsteigerung einerseits und Ausweitung der Anwendung andererseits. Das dritte Genus ist nicht mehr das Genus der Unbelebtheit, es assoziiert sich ebenso mit dem Gegenteil.

- Neutralisierung beschränkt sich nicht auf weibliche Personen (wie in westdeutschen und schweizerdeutschen Dialekten), sondern umfasst gleicherweise männliche Personen. Das 
ganze Feld der Personen-Pronominalisierung ist im Begriff, dem Neutrum zuzufallen.

- Dennoch sind traditionelle Zuteilungs-Regelungen (noch) nicht ausser Kraft gesetzt. Sie gehen einher mit radikalen Neuerungen. Das dreiteilige System bleibt dabei im Grossen und Ganzen bestehen. Dies erlaubt, Genus flexibel zuzuteilen; seine Anwendung bringt grammatische und/oder pragmatische, sich kreuzende Kongruenz- und InkongruenzPhänomene hervor.

- Bei Sachbezeichnungen ändert sich die Genus-Kongruenz nicht, bei Tieren nur in Ausnahmen (Haustiere im Neutrum).

- Die vom Neutrum beherrschte postverbale Subjektsenklise drängt sich massiv in die Verbalmorphologie ein und verändert sie von Grund auf. Durch Reanalyse und Grammatikalisierung gehen die neutralen Klitika in synthetisch-komprimierte Verbalendungen über.

- Seit der zweiten Hälfte des letzten Jahrhunderts verzeichnet die Neutralisierung von Personen durch Übergeneralisierung einen rasant ansteigenden Zuwachs; sie ist aber seit langem im System angelegt und lässt sich, wenn auch erst in Spuren, schon zu Beginn des 19. Jahrhunderts nachweisen.

\section{Literaturverzeichnis}

Bauen, Marco (1978): Sprachgemischter Mundartausdruck in Rimella (Valsesia, Piemont). Zur Syntax eines südwalserischen Dialekts im Spannungsfeld der italienischen Landes- und Kultursprache. Bern/Stuttgart, Haupt. (= Sprache und Dichtung. Neue Folge 28.)

Baumgartner, Gerda/Christen, Helen (2017): „Dr Hansjakobli und ds Babettli - Über die Geschlechtstypik diminuierter Rufnamen in der Deutschschweiz“. In: Reisig, Martin/Spiess, Constanze (eds.): Sprache und Geschlecht. Band 2. Duisburg: 111-145. (=Osnabrücker Beiträge zur Sprachtheorie 91).

Bucheli Berger, Claudia (2006): „Syntaktische Raumbilder im Höchstalemannischen“. In: Klausmann, Hubert (ed.): Raumstrukturen im Alemannischen. Graz-Feldkirch, Wolfgang Neugebauer Verlag: 91-96.

Bucheli Berger, Claudia (2019): „Verschwindet das Pronomen es für eine erwachsene Person aus den schweizerdeutschen Dialekten?“ In: Nievergelt, Andreas/Rübekeil, Ludwig (eds.): Raum und Sprache. Heidelberg, Universitätsverlag Winter: 313-327. (=Festschrift für Elvira Glaser).

Busley, Simone/Fritzinger, Julia (2018): „Em Stefanie sei Mann - Frauen im Neutrum“. In: Nübling, Damaris/Hirschauer, Stefan (eds.): Namen und Geschlechter-Studien zum onymischen Un/doing Gender. Berlin/Boston, De Gruyter: 191-212.

Busley, Simone/Fritzinger, Julia (2020): „De Lena sein Traum - Soziopragmatisch motivierte Genusvariabilität weiblicher Rufnamen“. In: Kempf, Luisa/Nübling, Damaris/Schmuck, Mirjam (eds.): Linguistik der Eigennamen. Berlin/München/Boston, De Gruyter: 347-376.

Christen, Helen (1998): „Die Mutti oder das Mutti, die Rita oder das Rita? Über Besonderheiten der Genuszuweisung bei Personen- und Verwandtschaftsnamen in schweizerdeutschten Dialekten“. In: Schnyder, André et al. (eds.): „Ist mir getroumet mîn leben“? Vom Träumen und vom Anderssein. Göppingen, Kümmerle Verlag: 267-281. (=Festschrift für Karl-Ernst Geith). 
Cigni, Costanza (2003): „Le formazioni diminutive nei dialetti delle comunità walser in Italia“. In : Fazzini, Elisabetta (Hg.): I dialetti walser in Italia: Contatto linguistico e scambi interculturali. Alessandria, Edizioni dell'Orso: 31-77. (= Studi Alemannici II).

Dal Negro, Silvia (2004): The Decay of a Language. The Case of a German Dialect in the Italian Alps. Bern, Peter Lang.

Fleischer, Jürg (2007): „Zur Herkunft des flektierten prädikativen Adjektivs im Höchstalemannischen“. In: Zeitschrift für Dialektologie und Linguistik 74: 196-240.

Frei, Gertrud (1970): Walserdeutsch in Saley: wortinhaltliche Untersuchung zu Mundart und Weltsicht der altertümlichen Siedlung Salecchio/Saley (Antigoriotal). Bern/Stuttgart, Haupt. (= Sprache und Dichtung Neue Folge 18.).

Gerstner-Hirzel, Emily (1979): Aus der Volksüberlieferung von Bosco Gurin. Sagen, Berichte und Meinungen. Märchen und Schwänke. Basel, Verlag G. Krebs. (= Schriften der Schweizerischen Gesellschaft für Volkskunde 63).

Giordani, Giovanni (1927): La colonia tedesca di Alagna-Valsesia e il suo dialetto. IIa edizione. Varallo Sesia, G.L.Testa Unione Tipografica Valsesiana. [1. Aufl. 1891. Nachdruck Bologna 1994, Libreria Alpina Editrice.]

Glaser, Elvira (2008). „Syntaktische Raumbilder“. In: Ernst, Peter/Patocka, Franz (Hg.): Dialektgeographie der Zukunft. Akten des 2. Kongresses der Internationalen Gesellschaft für Dialektologie des Deutschen (IGDD) am Institut für Germanistik der Universität Wien, 20. bis 23. September 2006. Stuttgart, Franz Steiner: 85-111.

Gysling, Fritz/Hotzenköcherle, Rudolf (1952): Walser Dialekte in Oberitalien in Text und Ton. Frauenfeld, Huber.

Id. = Schweizerisches Idiotikon. Wörterbuch der schweizerdeutschen Sprache. (1881-). Frauenfeld, Huber/Basel, Schwabe.

Keller, Hans-Erich (1975): „Ennetbirgische Walsertexte aus dem Beginn des 19. Jahrhunderts““. In: Semasia 2: 97-165.

Klein, Andreas/Nübling, Damaris (2019): ,,Was ist es mit diesem grammatisch ungeheuerlichen ,ihns'?' Zu Form und Funktion von alem. ääs, ihns und lux. hatt“. Linguistik Online 98(5), 51-76. doi.org/10.13092/lo.98.5929 [4.1.2021].

Ködel, Sven (2014): Die Enquête Coquebert de Montbret (1806-1812). Die Sprachen und Dialekte Frankreichs und die Wahrnehmung der französischen Sprachlandschaft während des Ersten Kaiserreichs. Bamberg, University of Bamberg Press. (= Bamberger Beiträge zur Linguistik 8).

Kolmer, Agnes (2009): „Man ist beeinflussbar. Zur Veränderlichkeit unpersönlicher Konstruktionen im Sprachkontakt“". In: Scholze, Lenka/Wiemer, Björn (Hg.): Von Zuständen, Dynamik und Veränderung bei Pygmäen und Giganten. Bochum, Brockmeyer: 229-244. (=Festschrift für Walter Breu).

Kolmer, Agnes (2012): Pronomen und Pronominalklitika im Cimbro. Untersuchungen zum grammatischen Wandel einer deutschen Minderheitensprache in romanischer Umgebung. Stuttgart, Franz Steiner. (=Zeitschrift für Dialektologie und Linguistik. Beihefte 150).

Nübling, Damaris (1992): Klitika im Deutschen. Schriftsprache, Umgangssprache, alemannische Dialekte. Tübingen, Gunter Narr. (= ScriptOralia 42). 
Nübling, Damaris (2017): „Funktionen neutraler Genuszuweisung bei Personennamen und Personenbezeichnungen im germanischen Vergleich“. In: Helmbrecht, Johannes/Nübling, Damaris/Schlücker, Barbara (Hg.): Namengrammatik. Hamburg: 173-211. (=Linguistische Berichte, Sonderheft 23)

Nübling, Damaris/Busley, Simone/Drenda, Juliane (2013): „Dat Anna und s Eva - Neutrale Frauenrufnamen in deutschen Dialekten und im Luxemburgischen zwischen pragmatischer und semantischer Genuszuweisung“. In: Zeitschrift für Dialektologie und Linguistik 80/2: 152-196.

Perinetto, Renato (1981): Eischemer's Büjie. Issime, unveröffentliches Typoskript.

Schott, Albert (1842): Die deutschen Colonien in Piemont. Ein Beitrag zur Geschichte der Alpen. Stuttgart und Tübingen, Cotta.

SDS = Sprachatlas der deutschen Schweiz (1962-1997). Band III (1975): Formengeographie. Bern, Francke.

Serianni, Luca (1989): Grammatica italiana. Torino, UTET Libreria.

Stalder, Franz Joseph (1819): Die Landessprachen der Schweiz oder Schweizerische Dialektologie. Mit kritischen Sprachbemerkungen beleuchtet, nebst der Gleichnisrede von dem verlorenen Sohne in allen Schweizermundarten. Aarau, Sauerländer.

Szadrowsky, Manfred (1930): „Zur hochalemannischen Syntax. III“. In: Beiträge zur Geschichte der deutschen Sprache und Literatur (PBB) 54: 281-293.

Waibel, Max (1985): Die volkstümliche Überlieferung in der Walserkolonie Macugnaga (Provinz Novara). Basel, Verlag G. Krebs. (= Schriften der Schweizerischen Gesellschaft für Volkskunde 70).

Wipf, Elisa (1910): Die Mundart von Visperterminen im Wallis. Frauenfeld: Huber. (= Beiträge zur Schweizerdeutschen Grammatik II).

Zürrer, Peter (1975): Wortfelder in der Mundart von Gressoney. Ein Beitrag zur Kenntnis der norditalienischen Walser-Mundarten. Frauenfeld: Huber. (= Beiträge zur schweizerdeutschen Mundartforschung 21).

Zürrer, Peter (1982): Wörterbuch der Mundart von Gressoney. Mit einer Einführung in die Sprachsituation und einem grammatischen Abriss. Frauenfeld: Huber. (=Beiträge zur schweizerdeutschen Mundartforschung 24).

Zürrer, Peter (1999): Sprachinseldialekte. Walserdeutsch im Aostatal (Italien). Aarau/Frankfurt am Main/Salzburg): Sauerländer. (= Reihe Sprachlandschaft 23).

Zürrer, Peter (2009): Sprachkontakt in Walser Dialekten. Gressoney und Issime im Aostatal (Italien). Stuttgart: Franz Steiner. (=Zeitschrift für Dialektologie und Linguistik. Beihefte 137).

Zürrer, Peter (2017): „L'inchiesta linguistica di Coquebert de Montbret nel Primo Impero (1806-1812). Progetto di un'edizione dei documenti relativi alle isole linguistiche dell'Italia settentrionale“. Bollettino dell'Atlante Linguistico Italiano. III Serie - Dispensa N. 41. Torino: $129-134$. 